\title{
Affective evaluations of objects are influenced by observed gaze direction and emotional expression
}

\author{
Andrew P. Bayliss ${ }^{\mathrm{a}, *}$, Alexandra Frischen ${ }^{\mathrm{b}}$, Mark J. Fenske ${ }^{\mathrm{c}}$, \\ Steven P. Tipper ${ }^{\text {a }}$ \\ a School of Psychology, University of Wales, Bangor, UK \\ ${ }^{\mathrm{b}}$ Department of Psychology, York University, Toronto, Ont., Canada \\ ${ }^{\mathrm{c}}$ MGH-Martinos Center for Biomedical Imaging, Harvard Medical School, Charlestown, MA, USA
}

Received 26 May 2006; revised 12 July 2006; accepted 15 July 2006

\begin{abstract}
Gaze direction signals another person's focus of interest. Facial expressions convey information about their mental state. Appropriate responses to these signals should reflect their combined influence, yet current evidence suggests that gaze-cueing effects for objects near an observed face are not modulated by its emotional expression. Here, we extend the investigation of perceived gaze direction and emotional expression by considering their combined influence on affective judgments. While traditional response-time measures revealed equal gaze-cueing effects for happy and disgust faces, affective evaluations critically depended on the combined product of gaze and emotion. Target objects looked at with a happy expression were liked more than objects looked at with a disgust expression. Objects not looked at were rated equally for both expressions. Our results demonstrate that facial expression does modulate the way that observers utilize gaze cues: Objects attended by others are evaluated according to the valence of their facial expression.
\end{abstract}

(c) 2006 Elsevier B.V. All rights reserved.

\footnotetext{
* Corresponding author. Tel.: +4401248 388367.

E-mail address: a.bayliss@bangor.ac.uk (A.P. Bayliss).
}

0010-0277/\$ - see front matter (c) 2006 Elsevier B.V. All rights reserved. doi:10.1016/j.cognition.2006.07.012 enced ..., Cognition (2006), doi:10.1016/j.cognition.2006.07.012. 
Observed gaze direction signals the focus of another person's interest. Hence, it is beneficial to shift one's attention to the same object looked at by another person. This phenomenon is known as "joint attention" (Baron-Cohen, 1995; Moore \& Dunham, 1995). Shifting attention to the direction of another's gaze results in faster responses to objects that appear in gazed-at, relative to other, locations (e.g., Bayliss, di Pellegrino, \& Tipper, 2004; Driver et al., 1999; Friesen \& Kingstone, 1998; Frischen \& Tipper, 2004; Langton \& Bruce, 1999).

Observing another's gaze direction also impacts arousal (Nichols \& Champness, 1971), and can modulate personality judgments of the observed faces (Bayliss \& Tipper, 2006; Macrae, Hood, Milne, Rowe, \& Mason, 2002; Mason, Tatkow, \& Macrae, 2005). Brain areas involved in encoding gaze direction and emotion perception are heavily interconnected and show interactions in activation (e.g., Adams, Gordon, Baird, Ambady, \& Kleck, 2003; Hoffman \& Haxby, 2000). Thus, it seems reasonable to expect behavioural responses to faces that differ in the emotion they express to also depend on where each face looks, and vice versa (e.g., Ganel, Goshen-Gottstein, \& Goodale, 2005). Indeed, the speed of recognizing expressed emotion and the consistency in attributing emotional traits for observed faces have recently been shown to critically depend on the joint contributions of both gaze direction and expressed emotion (Adams \& Kleck, 2003, 2005).

Given these results, it is surprising that gaze-dependent shifts of attention are not strongly affected by the observed facial expression of emotion. Hietanen \& Leppanen (2003), for example, found no evidence that happy, fearful, angry, or affectively neutral expressions yield differential effects of gaze direction on attention. Mathews, Fox, Yiend, \& Calder (2003) found enhanced gaze-cueing effects with fearful (versus neutral) faces, but only in a subset of their sample with heightened levels of anxiety (see also Tipples, 2006). Thus, the current evidence suggests that the interaction between observed gaze direction and emotion might be limited to only impact responses to the observed faces, but not to nearby objects (i.e., targets).

However, we propose that whether gaze direction and emotional expression interact is not determined by whether responses are made to the observed face or to nearby objects, but depends instead on whether the response requires affective evaluation. The key to this proposal is the fact that both gaze direction and emotional expression can indicate whether nearby objects are liked or disliked. People preferentially look at objects they like (Shimojo, Simion, Shimojo, \& Scheier, 2003), and their encounters with liked and disliked objects elicit very different emotional responses. Furthermore, such responses are thought to be mediated, respectively, by distinct neural systems (e.g., Cacioppo \& Gardner, 1999; Davidson, 1995; Davidson \& Irwin, 1999): an approach system using emotions that motivate appetitive 
behaviour (e.g., happiness) and an avoidance system using emotions to facilitate withdrawal from aversive stimuli (e.g., disgust).

Can similar emotional responses be activated by merely observing another person exhibiting object preference via their gaze direction? That is, if people understand that others preferentially look at objects they like (Baron-Cohen, Campbell, Karmilloff-Smith, Grant, \& Walker, 1995), then participants observing this looking behaviour might also like objects that are "cued" (i.e., looked at) by a stimulus face more than objects that the stimulus face looks away from. Indeed, using emotionally neutral stimulus faces, Bayliss, Paul, Cannon, \& Tipper (in press) found this precise pattern of results. However, whether this effect is a general effect of observing gaze-shifts toward objects or depends also upon the emotional expression of the face producing the gaze cue remains a very important question.

Hence, we developed a procedure examining gaze-cueing effects with a face that varied in emotional expression. Following previous work (e.g., Hietanen \& Leppanen, 2003), we predicted that the faster processing of gazed-at targets will not differ whether the face expresses happy or disgust emotions. However, in the same experiment, participants were asked to rate levels of liking of objects that were either gazed at (cued) or gazed away from (uncued). With this measure, a number of outcomes might be predicted.

Firstly, gazed-at target objects may be rated higher (i.e., liked more) than uncued targets, irrespective of emotional expression of the face. This would suggest that the attentional facilitation provided by the gaze cue boosts ratings of cued targets. Such attention-emotion effects have been found previously, but with attentional inhibition of ignored objects leading to lower ratings for ignored stimuli (Raymond, Fenske, \& Tavassoli, 2003). Alternately, the emotional expression of the face may override any gaze-related influence and alone determine rating responses. Targets appearing next to a disgusted face would thereby be liked less than those next to a happy face, irrespective of gaze direction. These two outcomes would indicate that gaze direction and emotional expression do not interact even when the behavioural response requires affective evaluation.

The final possibility is that observed gaze direction and emotional expression dynamically interact to influence ratings of the object of a joint attention episode. That is, cued objects are expected to be liked more when the face has a happy expression, but liked less when the face expresses disgust. However, because "uncued" objects are not the attentional focus of the observed face (or the target of its expressed emotion), only medium ratings are expected, with no general effect of emotional expression.

Through this new approach, it is hoped that a resolution to previous paradoxical findings concerning the combined effects of observed facial emotion and gaze-direction for nearby objects can be achieved within one experimental procedure. Interactions between gaze and emotion for objects other than the observed face may not be detected via traditional reaction time measures, but might be detected when measuring subsequent affective responses to these objects. 


\section{Experiment 1}

\subsection{Method}

\subsubsection{Participants}

Twenty-six adult volunteers were recruited from the School of Psychology at the University of Wales, Bangor (mean age $=19.3$ years, $S D=1.31$; two males). All had normal or corrected-to-normal vision, gave informed consent, and received course credit for participation.

\subsubsection{Apparatus and stimuli}

Stimulus presentation and data collection were controlled on a PC with millisecond timing using E-Prime (www.pstnet.com/eprime). Stimuli were presented $60 \mathrm{~cm}$ away (ensured using a chinrest) from the participant on a computer screen. Photographs of a female face $\left(14.9 \times 10.4^{\circ}\right)$ displaying either a moderately happy, moderately disgusted, or a neutral expression were used, looking left, right, or straight ahead were used (eyes: $1.91 \times 0.57^{\circ}$; pupils: 0.57 . The target stimuli consisted of 36 images of household items collected from the internet; 18 were 'garage' items (e.g., screwdriver, pliers, saw, etc.), 18 were "kitchen" items (e.g., a kettle, cutlery, a saucepan). The hue of the main section of each object was digitally manipulated, so the object could appear in red, blue, green, or yellow. Thus, there were a total of 144 possible target stimuli that could appear in their original orientation, or flipped about the vertical axis. The targets were presented $13.1^{\circ}$ from the centre of the screen. Targets varied between $3.43^{\circ}$ and $9.93^{\circ}$ in width, and between $1.43^{\circ}$ and $7.03^{\circ}$ in height. Two grey boxes $\left(10.9 \times 8.06^{\circ}\right)$ indicated target positions, on either side of the face. For the recording of preference ratings of the stimuli, a chart was presented, with the message "How much did you like that object?" at the top of the screen, with a column of numbers from 9 to 1 , headed by the words at the top, "Like very much," and with the words "Don't like at all" at the bottom of the screen.

\subsubsection{Design}

The factor "cueing" was whether the target was looked at (cued) or looked away from (uncued). The factor "emotion" was whether the face's expression changed from neutral to either happy or disgust prior to target onset. For each object, its colour, cueing status, and paired expression were determined randomly for each participant. Each object of a particular colour therefore appeared three times as a cued or uncued object with the same facial expression in a randomly determined location (left or right) and in a random orientation (normal or flipped).

\subsubsection{Procedure}

Participants were asked to ignore the uninformative gaze cue, and respond to the target as quickly as possible while maintaining fixation. Each trial started with a 600ms fixation cross. The face would then appear, with eyes gazing straight ahead for $1500 \mathrm{~ms}$, followed by the eyes shifting to the left or right and $250 \mathrm{~ms}$ later, the facial 


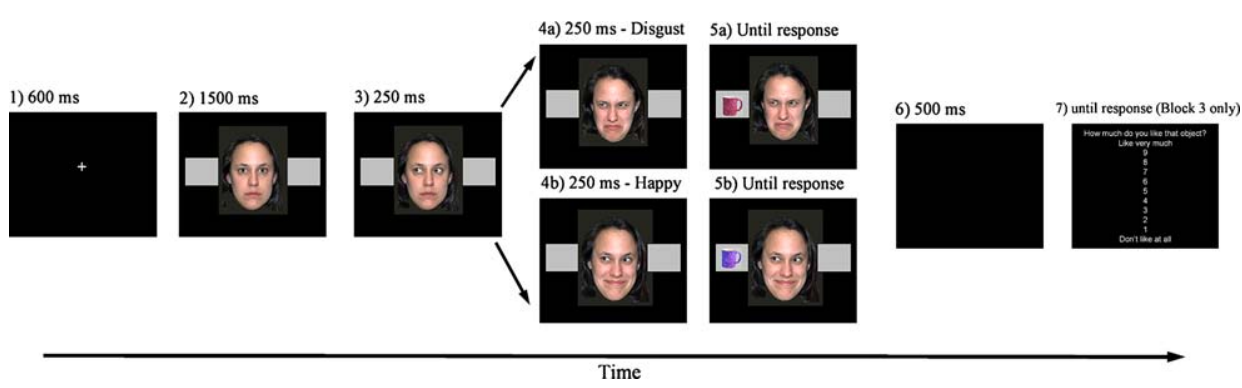

Fig. 1. Illustration of an experimental trial. Here, the face looks left (Box 3), validly cueing the eventual target location (a "cued" trial). Before the target appears, the face's expression changes to either disgusted (Box 4a), or to happy (Box 4b). Each individual target object appeared three times, consistently paired with either a disgusted, or happy face, and consistently appeared in a cued or uncued position. The targets were rated only in the final block, immediately after their final (3rd) appearance in the session (Box 7).

expression changing from neutral to happy or disgusted. The target appeared $250 \mathrm{~ms}$ later, remaining visible until response or until $2500 \mathrm{~ms}$ elapsed. Participants used the "h" and "spacebar" keys to respond to the category of the target object. Whether "h" corresponded to "Garage" or "Kitchen" items was counterbalanced between participants. At response, a tone was sounded to give feedback on performance (a "bell" for correct and a "buzzer" for incorrect/timeout). Finally, a 500-ms blank interval preceded the next trial (Fig. 1). After 10 practice trials, using the same cue faces, but different target objects, participants completed three blocks of 144 trials where each target was viewed once in each block. Repeating each object multiple times in this way was designed to ensure robust encoding of items under the same cueing and emotion conditions prior to their affective evaluation.

In the third block, the procedure changed. Participants were now informed that following their target categorization response, a blank screen would be presented $(500 \mathrm{~ms})$ followed by a rating screen (Fig. 1, Box 7). They were required to rate the item they had just responded to, such that higher scores were given to objects they liked more. They were told to verbally state a number from 1 to 9 , to be recorded by the experimenter. After their liking rating, another blank screen (500 ms) preceded the next trial. In total, therefore, participants completed 432 trials of the gazecueing procedure, being exposed three times to 36 targets looked at by a happy face, 36 targets that a happy face had looked away from, 36 targets looked at by a disgusted face, and 36 targets that had been looked away from by a disgusted face. Only in the final block, after seeing each object being either consistently cued or uncued by a disgusted or happy face three times, were these objects rated by the participants.

\subsection{Results and discussion}

\subsubsection{Gaze cueing}

Percent errors (4.8\% of trials) were submitted to a within-subjects ANOVA (an alpha of .05 was used for tests of statistical significance), with "cueing" and enced ..., Cognition (2006), doi:10.1016/j.cognition.2006.07.012. 
"emotion" as factors. Neither main effects nor the interaction approached significance, $F<1$. Correct responses contributed to the median RTs for each subject, in each condition, which were submitted to another ANOVA (Fig. 2). A significant main effect of "cueing" was found, $F(1,25)=16.8, \quad M S E=572.8, \quad p<.001$, $\eta_{p}^{2}=.402$, with quicker responses when the target was looked at by the face (cued trials $=663 \mathrm{~ms})$ than when the target was uncued $(682 \mathrm{~ms})$. Neither the main effect of emotion nor the interaction approached significance, $F<1.07, p>.31$. Hence, the disgust and happy faces produced almost identical amounts of attentional cueing (19 and $20 \mathrm{~ms}$, respectively). These results are consistent with the conclusions of
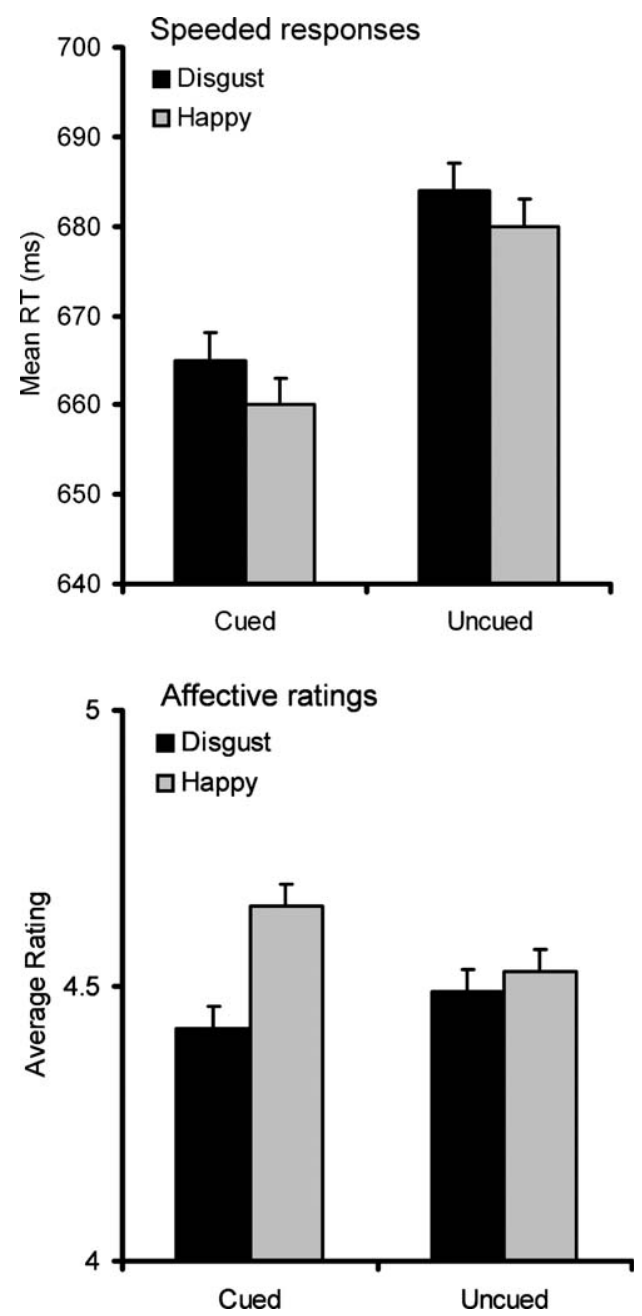

Fig. 2. Mean reaction times (upper panel) and mean ratings (lower panel) for targets appearing in each cueing condition. Error bars represent standard errors of the means based on the procedure suggested by Loftus and Masson (1994) for within-subjects designs.

Please cite this article as: Andrew P. Bayliss et al., Affective evaluations of objects are influenced ..., Cognition (2006), doi:10.1016/j.cognition.2006.07.012. 
Hietanen \& Leppanen (2003) that facial expression does not influence the strength of the shift of attention following a gaze-cue.

\subsubsection{Object ratings}

Ratings for target objects that were also correctly categorized in the final block of the experiment contributed to cell means that were submitted to a within-subjects ANOVA with "emotion" and "cueing" as factors. Firstly, the main effect of "emotion" was significant, $F(1,25)=6.32, M S E=.069, p=.019, \eta_{p}^{2}=.202$, due to participants assigning a higher rating to items appearing alongside a happy expression, compared to a disgusted expression (4.59 vs. 4.46 on the $1-9$ scale, respectively). Overall, the effect of the face looking at or away from the target ("cueing") was non-significant, $F(1,25)<1, M S E=.031$. However, both these effects were qualified by a significant interaction, $F(1,25)=5.94, M S E=.038$, $p=.022, \eta_{p}^{2}=.192$, whereby happy-cued items $(M=4.65)$ were liked more than disgust-cued items $(M=4.42)$, with no apparent difference between the ratings of uncued items (rated 4.53 and 4.49, respectively, see Fig. 2). Planned contrasts confirmed that disgust-cued items were rated lower than the happy-cued items $t(25)=3.19, p=.004, d=.293$, whereas the two types of uncued targets were not rated differently to each other $t(25)=.623, p=.539, d=.046$. Further contrasts revealed that the happy-cued targets were rated significantly higher than the happy-uncued targets, $t(25)=2.13, p=.043, d=.150$, while the trend for disgust-cued targets to be liked less than disgust-uncued targets failed to reach significance, $t(25)=-1.44, p=.162, d=.090$. Hence, it appears that while the emotional expression of a gazing face has little impact on attention, it can strongly influence affective evaluation of the target object, but only when the target is "looked at."

\section{Experiment 2}

While Experiment 1 compared "looked-at" targets with "looked-away-from" targets, neither of these conditions involve an interaction between emotional expression and the most powerful gaze behaviour-gaze directed toward the participant. Hence, a second experiment was conducted, whereby the eyes in the face remained looking straight ahead (for $1750 \mathrm{~ms}$ ) on all trials while the emotion changed $(250 \mathrm{~ms}$ before target onset). Twenty-six participants volunteered for the study (eight males, mean age $=20.1$ years, $S D=2.79$ ). The stimuli, design, and procedure were identical to Experiment 1, except that the factor "cueing" was dropped, since the eyes stayed staring straight ahead. Paired $t$ tests showed that neither errors $(5.72 \%$ on average), RTs (735 vs. $734 \mathrm{~ms})$, nor ratings (4.64 vs. 4.59 for targets appearing next to "happy" and "disgust" expressions, respectively) were significantly affected by emotional expression $(t<.62, p>.56, d<.1)$. Hence, if gaze is not directed towards the object, as in this experiment and the uncued condition of Experiment 1, emotional expression does not influence object ratings. 


\section{General discussion}

The results of this study are clear, and they resolve previous conflicts in the literature. When assessing people's level of liking of objects, it seems that there is an intimate and highly specific relationship between gaze direction and the emotion expressed by the face. Thus, when a face is seen to be looking at an object, the emotion expressed by the face determines how much the object is liked. Objects are liked more when the face looking at them is smiling than when expressing disgust. This influence of face emotion is not a general effect influencing objects in the environment, because there is no effect of happy or disgust emotions on objects that are not directly looked at. That is, only ratings of the object of joint attention are subject to modulation via emotional expression. Thus, when evaluating an object that is gazed at by another person, taking into account their emotional expression helps to appraise the significance of that object. On the other hand, the emotional expression of the person conveys little information about an object that is not looked at. Interestingly, when asked during casual debriefing, none of the participants indicated that either the face's gaze direction or emotional expression had influenced their ratings, asserting instead that they had made ratings based on the objects' usefulness and aesthetic appeal. This supports the possibility that the combined effects of observed gaze and emotion can be evoked implicitly.

Our data suggest that gaze cues can trigger two independent effects. This can be appreciated by just considering a participant's response when a face looks at an object. The participant is about $20 \mathrm{~ms}$ faster to respond to this object whether or not the face smiles or appears disgusted. In sharp contrast, participants provide quite different reports about how much they like the cued objects. Thus, the increase in processing fluency when categorizing the cued objects did not affect liking per se. ${ }^{1}$ This underscores the importance of the gaze-cue as a social signal, rather than just as an attentional cue, when it is used in conjunction with the emotion of the face to influence liking.

Different neural pathways might mediate the independent effects of gaze as an attentional cue from those as a social cue. The rapid and automatic orientation of attention to the location looked at is perhaps mediated by a pathway from the gaze processing system of the superior temporal sulcus (STS) to the attention movement systems of the intraparietal sulcus (Wicker, Michel, Henaff, \& Decety, 1998). However, there is clearly a second form of processing where the gaze and emotion expressed by others help us evaluate the potential value of objects in the environment. This processing is likely mediated by links between the STS and amygdala

\footnotetext{
${ }^{1}$ The fact that attentional facilitation did not boost ratings of cued objects in this paradigm is interesting. While this study was vastly different from that of Raymond et al. (2003), it is possible that only attentional inhibition can produce (negative) effects on affective ratings of objects, while attentional facilitation does not result in positive effects on ratings. Alternatively, the manner in which the attention is manipulated (gaze-cue, peripheral cue, volitional orienting) may be critical in determining interactions between attention and emotion.
}

Please cite this article as: Andrew P. Bayliss et al., Affective evaluations of objects are influenced ..., Cognition (2006), doi:10.1016/j.cognition.2006.07.012. 
(e.g., Adams et al., 2003) and it enables vicarious learning, which is a major advantage for social animals. Such learning appears to emerge early in life, as previous developmental work suggests that infants are sensitive to such cues from the age of about 1 year (Mumme \& Fernald, 2003; Repacholi, 1998). Hence in highly complex environments, gaze and emotion signals can identify which object is relevant, and provide the cues as to whether it is good (approach) or bad (avoid).

\section{Acknowledgments}

This work was supported by ESRC grants to A.B. (PTA-026-27-0980) and S.T. (RES-000-23-0429). The MacBrain Face Set was developed by Nim Tottenham (tott0006@tc.umn.edu) through John D. and Catherine T. MacArthur Foundation support.

\section{References}

Adams, R. B., Gordon, H. L., Baird, A. A., Ambady, N., \& Kleck, R. E. (2003). Effects of gaze on amygdala sensitivity to anger and fear faces. Science, 300, 1536.

Adams, R. B., \& Kleck, R. E. (2003). Perceived gaze direction and the processing of facial displays of emotion. Psychological Science, 14(6), 644-647.

Adams, R. B., \& Kleck, R. E. (2005). Effects of direct and averted gaze on the perception of facially communicated emotion. Emotion, 5(1), 3-11.

Baron-Cohen, S. (1995). The eye direction detector (EDD) and the shared attention mechanism (SAM): Two cases for evolutionary psychology. In C. Moore \& P. J. Dunham (Eds.), Joint attention: Its origins and role in development (pp. 41-59). Hillsdale, NJ: Lawrence Erlbaum.

Baron-Cohen, S., Campbell, R., Karmilloff-Smith, R., Grant, J., \& Walker, J. (1995). Are children with autism blind to the mentalistic significance of the eyes?. British Journal of Developmental Psychology 13, 379-398.

Bayliss, A. P., di Pellegrino, G., \& Tipper, S. P. (2004). Orienting to the direction of observed eye-gaze is head-centred. Cognition, 94(1), B1-B10.

Bayliss, A. P., Paul, M. A., Cannon, P. R., Tipper, S. P. (in press). Gaze cueing and affective judgments of objects: I like what you look at. Psychonomic Bulletin \& Review.

Bayliss, A. P., \& Tipper, S. P. (2006). Predictive gaze cues and personality judgments: Should eye trust you?. Psychological Science 17(6), 514-520.

Cacioppo, J. T., \& Gardner, W. L. (1999). Emotions. Annual Review of Psychology, 50, 191-214.

Davidson, R. J. (1995). Cerebral asymmetry, emotion, and affective style. In R. J. Davidson \& K. Hugdahl (Eds.), Brain asymmetry. Cambridge, MA: MIT Press.

Davidson, R. J., \& Irwin, W. (1999). The functional neuroanatomy of emotional affective style. Trends in Cognitive Sciences, 3(1), 11-21.

Driver, J., Davis, G., Ricciardelli, P., Kidd, P., Maxwell, E., \& Baron-Cohen, S. (1999). Gaze perception triggers reflexive visuospatial orienting. Visual Cognition, 6(5), 509-540.

Friesen, C. K., \& Kingstone, A. (1998). The eyes have it! Reflexive orienting is triggered by nonpredictive gaze. Psychonomic Bulletin \& Review, 5(3), 490-495.

Frischen, A., \& Tipper, S. P. (2004). Orienting attention via observed gaze shift evokes longer-term inhibitory effects: Implications for social interactions, attention and memory. Journal of Experimental Psychology, 133(4), 516-533.

Ganel, T., Goshen-Gottstein, Y., \& Goodale, M. A. (2005). Interactions between the processing of gaze direction and facial expression. Vision Research, 45(9), 1191-1200. enced ..., Cognition (2006), doi:10.1016/j.cognition.2006.07.012. 
Hietanen, J. K., \& Leppanen, J. M. (2003). Does facial expression affect attention orienting by gaze direction cues?. Journal of Experimental Psychology 29(6), 1228-1243.

Hoffman, E. A., \& Haxby, J. V. (2000). Distinct representations of eye gaze and identity in the distributed human neural system for face perception. Nature Neuroscience, 3(1), 80-84.

Langton, S. R. H., \& Bruce, V. (1999). Reflexive visual orienting in response to the social attention of others. Visual Cognition, 6(5), 541-567.

Loftus, G. R., \& Masson, M. E. J. (1994). Using confidence intervals in within subjects designs. Psychonomic Bulletin \& Review, 1, 476-490.

Macrae, C. N., Hood, B. M., Milne, A. B., Rowe, A. C., \& Mason, M. F. (2002). Are you looking at me? Eye gaze and person perception. Psychological Science, 13(5), 460-464.

Mathews, A., Fox, E., Yiend, J., \& Calder, A. (2003). The face of fear: Effects of eye gaze and emotion on visual attention. Visual Cognition, 10(7), 823-835.

Mason, M. F., Tatkow, E. P., \& Macrae, C. N. (2005). The look of love: Gaze shifts and person perception. Psychological Science, 16(3), 236-239.

Moore, C., \& Dunham, P. J. (Eds.). (1995). Joint attention: Its origins and role in development. Hove, UK: Lawrence Erlbaum.

Mumme, D. L., \& Fernald, A. (2003). The infant as onlooker: Learning from emotional reactions observed in a television scenario. Child Development, 74(1), 221-237.

Nichols, K. A., \& Champness, B. G. (1971). Eye gaze and the GSR. Journal of Experimental Social Psychology, 7, 623-626.

Raymond, J. E., Fenske, M. J., \& Tavassoli, N. (2003). Selective attention determines emotional responses to novel stimuli. Psychological Science, 14, 537-542.

Repacholi, B. M. (1998). Infants' use of attentional cues to identify the referent of another person's emotional expression. Developmental Psychology, 34, 1017-1025.

Shimojo, S., Simion, C., Shimojo, E., \& Scheier, C. (2003). Gaze bias both reflects and influences preference. Nature Neuroscience, 6(12), 1317-1322.

Tipples, J. (2006). Fear and fearfulness potentiate automatic orienting to eye gaze. Cognition and Emotion, 20, 309-320.

Wicker, B., Michel, F., Henaff, M., \& Decety, J. (1998). Brain regions involved in the perception of gaze: A PET study. NeuroImage, 8, 221-227. 